\title{
La base de données SYLVESTRE au service de la qualité et de l'expertise en radioécologie à l'IRSN
}

\author{
C. DUFFA ${ }^{1}$, C. ANTONELLI ${ }^{2}$, F. VRAY ${ }^{2}$, M.-J. MARANT ${ }^{3}$, G. SALAUN $^{2}$
}

(Manuscrit reçu le 3 octobre 2006, accepté le 7 janvier 2007)

RÉSUMÉ SYLVESTRE est une base de données de l'IRSN permettant la valorisation, l'exploitation et le stockage des données ayant trait aux mesures de radioactivité réalisées sur des échantillons de l'environnement. Constamment alimentée et améliorée depuis sa création en 1991, elle constitue aujourd'hui un outil essentiel au service de la qualité. Les utilisateurs ont un accès facile, via le réseau interne de l'IRSN, à l'interface développée afin de répondre au mieux aux différents besoins : introduction des données ayant trait aux prélèvements, aux préparations et aux mesures, exportations de pans de données à des fins de vérifications ou d'expertises. Le souci constant d'amélioration des fonctionnalités et de minimisation des saisies ou des recopies manuelles de données dans la base a conduit à mettre en place plusieurs outils ou programmes périphériques (tablettes numériques de terrain, étiquetages codes-barres des échantillons, automatisation de requêtes multicritères, ....).

ABSTRACT SYLVESTRE database for quality and radioecological expertise at IRSN.

SYLVESTRE is an IRSN database, allowing improvement and storage of data concerning radioactivity measurements on environmental samples. Continuously supplied and upgraded since its creation in 1991, it is now an essential tool for quality. It is also a precious tool used by experts. Users have an easy access to the dedicated interface through internal IRSN network. They can introduce data concerning sample collections, preparations and measurements, export sections of data for checking or assessment. Several peripheral tools or programs (numerical field tablets, bar-code sample labelling, multicriterion requests automation, ...) have been developed in order to improve the application and to minimize manual data captures or replicates.

Keywords: database / environmental samples / quality / radioecological assessment

\section{Introduction}

La base de données SYLVESTRE (SYstème Logique de Valorisation Exploitation et STockage des données sur la Radioactivité dans l'Environnement) est un outil développé à l'IRSN, dans le but de rassembler, sous forme informatique et sécurisée, l'ensemble des données relatives à l'acquisition de résultats de mesures

${ }^{1}$ IRSN/DEI/SESURE/LERCM, Port du Bregaillon, BP 330, 83507 La Seyne-sur-Mer, France.

2 IRSN/DEI/SESURE/LERCM, CE Cadarache, B.P. 3, 13115 St Paul-Lez-Durance, France.

${ }^{3}$ Communication \& Systèmes, Cité Grande Bastide, bâtiment 914, 13115 St Paul-Lez-Durance, France. 


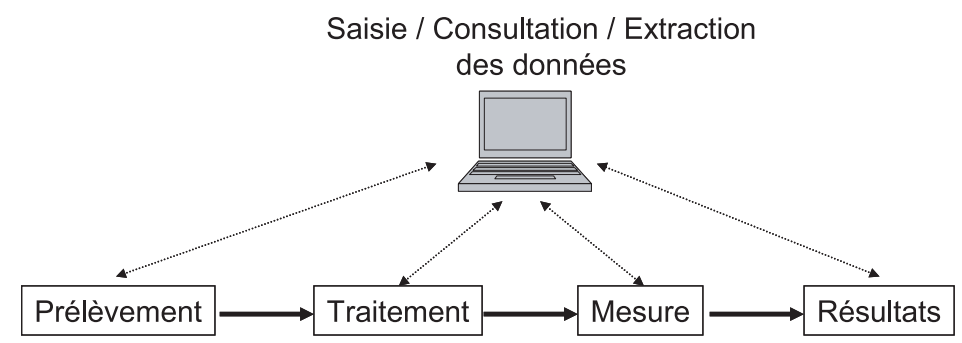

Figure 1 - SYLVESTRE au cæur du système de gestion et d'information sur les mesures de radioactivité dans l'environnement.

SYLVESTRE in the centre of the management and data on radioactivity in the environment system.

effectuées sur des échantillons prélevés dans l'environnement dans le cadre d'études et expertises radioécologiques. Depuis sa création en 1991, cette base de données n'a cessé d'évoluer tant sur le plan technique qu'au niveau de ses fonctionnalités, afin de répondre au mieux aux besoins des utilisateurs. L'objet de cet article est de présenter cet outil opérationnel, fruit de plusieurs années d'expérience, qui constitue actuellement un exemple dont pourraient s'inspirer d'autres organismes ou laboratoires.

À l'heure actuelle, SYLVESTRE présente deux aspects principaux :

- d'une part, une fonctionnalité d'intégration et de gestion des données relatives aux échantillons. Le suivi des actions de terrain et de préparation des échantillons en laboratoire est réalisé à l'aide de la base dans laquelle est renseignée chacune des étapes de la réalisation d'une campagne de mesure (Fig. 1). Ceci permet de répertorier les renseignements obtenus lors des actions d'identification des prélèvements, de traitement d'échantillons et de mesure radioactive ou autre ;

- d'autre part, une fonctionnalité de consultation par extractions sélectives, permettant notamment de dresser un état de la radioactivité par type d'échantillon, radionucléide, zone de prélèvement, date, ...

En 2006, près de 370000 résultats de mesures sont stockés dans cette base, auxquels s'ajoute l'ensemble des indications, qualitatives et/ou quantitatives, relatives à la gestion des échantillons ; environ 10000 résultats viennent s'ajouter chaque année au stock de données déjà acquises.

\section{Description technique}

SYLVESTRE est une base de données relationnelle Oracle ${ }^{\mathrm{TM}}$. Elle est installée sur un serveur de données, qui abrite les informations et gère les transactions des 
utilisateurs. Les utilisateurs consultent, alimentent et exploitent la base depuis n'importe quel poste IRSN via une interface Intranet développée en Java et Javascript. L'IHM SYLVESTRE, qui met en forme les données en provenance de la base, est implantée sur un autre serveur (serveur d'applications).

Le poste client est totalement banalisé. Les traitements s'effectuant sur les serveurs d'applications et de données, aucune installation complémentaire n'est nécessaire.

SYLVESTRE est évolutif. L'ajout ou la modification d'une partie de l'application n'entraîne pas un nouveau déploiement de celle-ci sur les postes clients : les évolutions sont immédiatement disponibles.

SYLVESTRE est sécurisé. En effet, exploitant une architecture trois tiers, le «client » n'a jamais directement accès au serveur de données (base de données Oracle) mais passe par le serveur d'applications.

\section{Gestion et stockage des données sur les prélèvements, traitements et mesures}

Dès lors que l'on décide de faire le diagnostic à la fois qualitatif et quantitatif de l'état radioécologique de l'environnement, il est nécessaire de prélever des échantillons représentatifs des différentes matrices d'intérêt. Ces échantillons doivent ensuite être préparés afin d'être analysés en laboratoire par des techniques qui peuvent différer selon les radionucléides recherchés. À chaque échantillon sont rattachées de nombreuses informations ayant trait à ces différentes étapes jusqu'à obtention des résultats d'analyse (Fig. 2). C'est l'ensemble de ces données qui sont enregistrées dans la base SYLVESTRE.

Chaque échantillon est identifié et codé à partir d'informations clés stockées dans la base : nature de l'échantillon, année de prélèvement, mission ou site étudié, numéro de l'échantillon et éventuellement numéro de fraction et numéro de traitement. Ces données, figurées en clair sur chaque échantillon, sont également intégrées dans un code-barres qui servira au suivi de l'échantillon.

Afin de minimiser les erreurs de recopie d'informations notées manuellement sur le terrain, le préleveur utilise des ardoises de terrain intégrant un GPS et une imprimante de code-barres qui permettent la saisie directe des informations (Fig. 3). Au retour de mission les fichiers de données sont importés informatiquement dans SYLVESTRE.

Outre cette fonction d'enregistrement de données, SYLVESTRE permet aussi la gestion des envois de lots d'échantillons pour analyse. Seuls les échantillons 

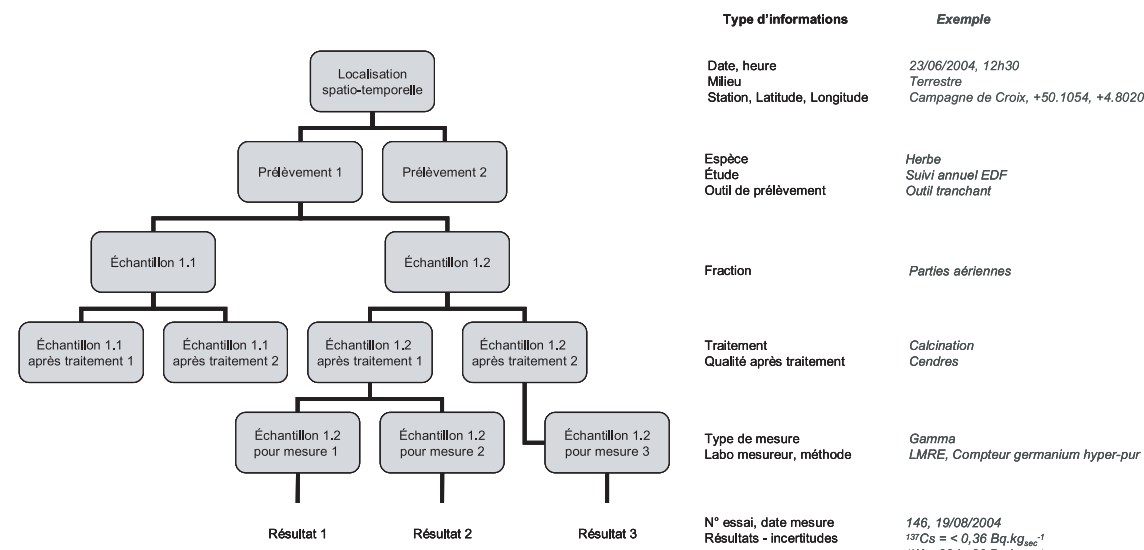

Figure 2 - Organigramme des informations enregistrées dans SYLVESTRE.

SYLVESTRE recorded data flow chart.

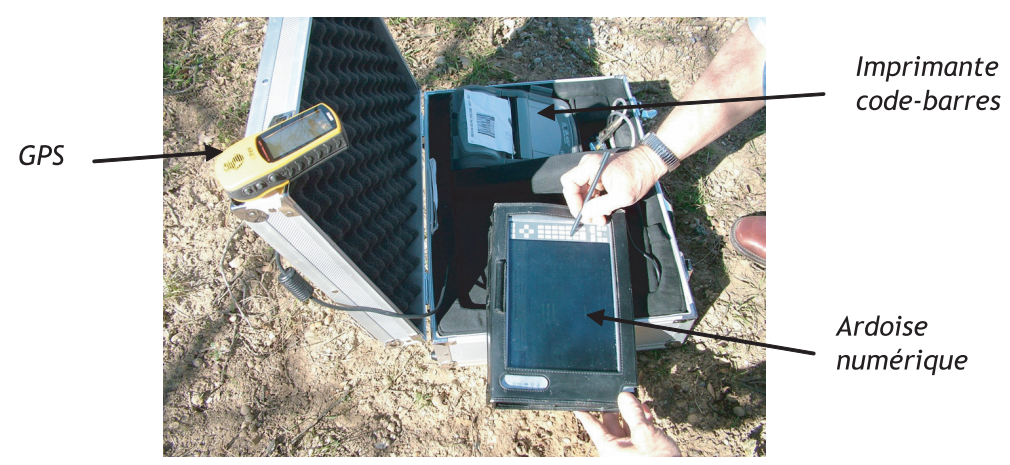

Figure 3 - Équipement permettant la saisie des informations relatives aux prélèvements.

Equipment for samples relative data input.

dont les données relatives au prélèvement et au traitement ont été validées par le chargé d'étude peuvent être intégrés dans un lot pour envoi vers les laboratoires de mesures.

Les résultats sont renvoyés par les laboratoires de mesures sous forme de fichiers informatiques à un correspondant unique qui les intègre dans la base directement. Ce système d'intégration de fichiers de données permet de s'affranchir des erreurs de saisie manuelles toujours possibles. 


\section{Support à l'expertise}

Plusieurs fonctions, parties intégrantes de l'application, ont été développées afin que SYLVESTRE ne soit pas seulement une base d'archivage de données, mais aussi un vrai support à l'expertise. Ainsi, la consultation des données est possible par différentes méthodes : pour chaque échantillon, il est possible d'éditer une fiche récapitulant l'ensemble des informations enregistrées (Fig. 4); un module de requêtes propre à l'applicatif SYLVESTRE permet l'extraction de pans de données et de résultats selon des critères définis par l'utilisateur. On pourra par exemple extraire tous les résultats de mesure de ${ }^{137} \mathrm{Cs}$ effectués autour d'une installation électronucléaire choisie. Ce « requêteur » a été conçu de manière à ce que l'utilisateur puisse choisir ses critères parmi des listes intelligibles, sans avoir à connaître la structure complexe de la base SYLVESTRE.

Enfin, plusieurs programmes complémentaires et périphériques à la base de données elle-même ont été développés. Ils permettent en particulier l'édition automatisée de tableaux descriptifs des prélèvements et des résultats de mesures acquis autour des sites nucléaires (Fig. 5), publiés notamment dans les rapports d'expertise réalisés pour le compte d'EDF (rapports annuels IRSN « Suivi radioécologique de l'environnement terrestre, aquatique continental et marin des centrales nucléaires françaises »). Leur utilisation offre une lisibilité et un gain de temps important lors de l'exploitation des données tout en garantissant la validité des résultats présentés, notamment des rapports d'activité usuels (ex : $\left.{ }^{238} \mathrm{Pu} /{ }^{239+240} \mathrm{Pu}\right)$ dont le calcul est automatisé.

\section{Conclusion}

La base SYLVESTRE regroupe actuellement les informations sur 48000 prélèvements effectués dans l'environnement français dans le cadre des missions d'expertises et d'études de l'IRSN : études radioécologiques autour des installations électronucléaires et programme d'observation OPERA (Observatoires PErmanents de la RAdioactivité dans l'environnement) en particulier. Les résultats d'environ 80000 mesures sont ainsi reportés dans SYLVESTRE.

Les évolutions techniques et structurelles nécessaires à la pérennité de cet outil sont réalisées sous la responsabilité d'un administrateur dans le cadre d'un contrat de maintenance. SYLVESTRE étant devenu un outil central et indispensable pour le travail des personnes en charge des prélèvements et des préparations d'échantillons, ces évolutions sont régulières et nécessaires, assurant ainsi une amélioration constante de la qualité et de la traçabilité des données. La mise en place récente de l'étiquetage code-barres des échantillons va dans ce sens. Son utilisation permettra à terme des échanges facilités et sécurisés avec les 


\begin{tabular}{|c|c|c|c|c|c|}
\hline \multicolumn{7}{|c|}{$\begin{array}{r}\text { FICHE ANALYSE } \\
\text { Milieu terrestre } \\
\text { LERCM-Cadarache }\end{array}$} \\
\hline \multicolumn{7}{|c|}{$\begin{array}{c}\text { Code Site/Mission } \\
\text { Date de prélèvement }\end{array}$} & $\begin{array}{c}\text { Heure de } \\
\text { préléve d'identification }\end{array}$ & Code de la station & Numéro de fraction \\
\hline $08 / 06 / 2004$ & $12: 00: 00$ & OPSPA (No 1641) & OAP & 10 & 1 \\
\hline & & La Parise, Forcalquier & & $\begin{array}{c}\text { Plantes aromatiques } \\
\text { Thym }\end{array}$ & Parties aérennes \\
\hline
\end{tabular}

Spectrométrie gamma (GAMMA ) effectuée par : Laboratoire de Mesure de la Radioactivité dans l'Environnement d'Orsay (LMRES) Quantitél Qualité analysée 15,0 unité cendres

Méthode d'analyse Germanium hyper pur (GEHP)

Date d'envoi en analyse $05 / 07 / 2004$

$\mathrm{N}^{\circ}$ chrono de l'envoi $\mathrm{C}-2004-211$

Responsable SALAUN

\begin{tabular}{|r|l|}
\hline $\mathbf{N}^{\circ}$ du lot (mesureur) & 128 \\
\hline $\mathbf{N}^{\circ}$ de la note de résultats & $04-885$ \\
\hline Date de réception des résultats & $20 / 09 / 2004$ \\
\hline $\mathbf{N}^{\circ}$ de la mesure & \\
\hline Date de la mesure & $21 / 07 / 2004$ \\
\hline
\end{tabular}

Rappel sur le traitement subi par l'échantillon :
\begin{tabular}{|l|l|l|l|}
\hline \multicolumn{1}{|c|}{ Traitement } & \multicolumn{1}{|c|}{ Libellé } & Caractéristiques & \multicolumn{1}{c|}{ Valeurs } \\
\hline 1: ETUVE & Séchage par étuvage & PFIPS & 2.267929 \\
& & TMAX & 80 \\
\hline $2: \mathrm{CALCl}$ & Calcination & PS/PC & 13.063946 \\
& & TMAX & 480 \\
\hline
\end{tabular}

\begin{tabular}{|c|c|c|c|c|c|}
\hline \multicolumn{7}{|c|}{ Les résultats ont été saisis dans SYLVESTRE le 24/09/2004 } \\
\hline Elément dosé & Unité & Résultat & Incertitude & Indicateur & Indice de validité \\
\hline Ac228 & BqKgS & 3,0619 & 0,6124 & & \\
\hline Ag110 & BqKgS & & 0,3827 & $<$ & \\
\hline Am241 & BqKgS & & 0,237294 & $<$ & \\
\hline Be7 & BqKgS & 173,7607 & 16,8402 & & \\
\hline Co57 & BqKgS & & 0,1072 & $<$ & \\
\hline C058 & BqKgS & & 0,3062 & $<$ & \\
\hline Co60 & BqKgS & & 0,2373 & $<$ & \\
\hline Cs134 & BqKgS & & 0,6889 & $<$ & \\
\hline Cs137 & BqKgS & 0,7731 & 0,1148 & & \\
\hline Eu154 & BqKgS & & 0,2067 & $<$ & \\
\hline K40 & BqKgS & 497,5526 & 53,5826 & & \\
\hline Mn54 & BqKgS & & 0,3062 & $<$ & \\
\hline Na22 & BqKgS & & 0,2526 & $<$ & \\
\hline Pb210 & BqKgS & 70,4228 & 8,4201 & & \\
\hline Rh106 & BqKgS & & 1,9137 & $<$ & \\
\hline Sb124 & BqKgS & & 0,3062 & $<$ & \\
\hline Sb125 & BqKgS & & 0,4593 & $<$ & \\
\hline Th234 & BqKgS & & 4,5928 & $<$ & \\
\hline
\end{tabular}

Figure 4 - Fiche de synthèse des informations ayant trait à la mesure gamma effectuées sur un échantillon de thym prélevé le 09/06/2004 dans le cadre des missions OPERA (Observatoires Permanents de la Radioactivité dans l'Environnement, programme d'observation IRSN).

Synthesis sheet on data related to gamma measurement carried on a thyme sample collected on the 06/09/2004 for OPERA program. 


\begin{tabular}{|c|c|c|c|c|c|c|c|}
\hline $\begin{array}{l}\text { Siluation par } \\
\text { rapportala } \\
\text { Centrale }\end{array}$ & Station & Nature & Espece & Fraction & $\begin{array}{c}\text { Date de } \\
\text { prekevement }\end{array}$ & Frais/sece & Secicendur \\
\hline Sur site & CNFE Dampieme, piérs 1.1 & Ean douce & Ean de nappe pluéádique & Extier & $2504 / 2001$ & - & - \\
\hline Sur site & CNFE Danpieme, piérs 1.1 & Ean douce & Ean de nappe pluéstique & Eaul filtrée à 0,22 micromètres & $30,08: 2001$ & - & - \\
\hline $1 . \mathrm{km}$ ONO & Danpiente en-Burly, femre Ruchires & Sols cultives & Sol de culture de plenichanm $0-22.0 \mathrm{~cm}$ & Diamtren inféneư à 2 mum & $26,09: 2001$ & 1,11 & - \\
\hline 1.km ONo & Dampieme-en-Eurby, ferme Ruchères & Céréales & $\begin{array}{c}\text { Mais } \\
\text { Zeanqus }\end{array}$ & Graine & 26,0925001 & 1,69 & 61,79 \\
\hline $1 . \mathrm{kmo}$ & Femre Les Mallerets, piemo NS & Eand douce & Ean de nappe pluéátique & Eau filitrée à 0,22 micromètres & 25.0452001 & - & - \\
\hline $1 \mathrm{kmo}$ & Feme Les Mallerets, piens NS & Ean doure & Ean de nappe pluéstique & Eaul filtrée à 0,22 micromètres & 30,082001 & - & - \\
\hline $2 \mathrm{kmE}$ & Lioner-sullias, Chemin de la Loire & Ean douce & Eau de nappe pluéádique & Entior & $24,04: 2001$ & - & - \\
\hline $5 \mathrm{~km} s \mathrm{O}$ & Saint-Florent & Mousses temiooles & 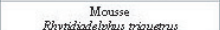 & Extier & $24,04 / 2001$ & 1,22 & - \\
\hline $9 \mathrm{kmsO}$ & Charkexy & Légumes & Salade non identifí̌e & Parties áninzmes & 29,0825001 & 21,37 & 4,01 \\
\hline $9 \mathrm{~km} \mathrm{NE}$ & Carrefour du poiner à la Demoiselle & Arbre feuilh & $\begin{array}{l}\text { Chêre pédonculé } \\
\text { Quercaus robur }\end{array}$ & Feuilles armuelles & $2808 / 2501$ & 2,39 & - \\
\hline $9 \mathrm{kmsO}$ & Charkery & Arbre feuilh & $\begin{array}{l}\text { Chêre pédconculé } \\
\text { Quereaus robur }\end{array}$ & Fenilles armuelles & 29.082501 & 2,31 & - \\
\hline $9 . \mathrm{kmNE}$ & Camefour du poinèr à la Demoiselle & Champigrons & $\begin{array}{l}\text { Bolet bai } \\
\text { Xerocomus badius }\end{array}$ & Parties á́riemes & $26,09: 2001$ & 12,83 & - \\
\hline $95 \mathrm{kmsE}$ & Gien & Sols cultives & Sol de culture de pleinchann $0-2 x .0 m$ & Dianìtre irféneưu à $2 x u m$ & $26,09 / 2001$ & 1,34 & - \\
\hline $95 \mathrm{kmSE}$ & Poilly-les-Gien, route de Gien & Céréales & $\begin{array}{l}\text { Mais } \\
\text { Zeamaqs }\end{array}$ & Graine & 26.092501 & 1,72 & 65,3 \\
\hline $25 \mathrm{kmSE}$ & Poilly-les-Gien & Volaille & Poule & Muscle & 29,0820202 & 3,74 & 17,06 \\
\hline 10.5 kan ONO & Saint:Pèn-sur-Loire & Ean douce & Ean d'imization & Eau filińé à 0,22 micromèthes & $26,09: 5001$ & - & - \\
\hline 11. lan SE & Gien & Légumes & Salade non identifí́e & Parties árinemes & 29,082501 & 22,71 & - \\
\hline 15. $\operatorname{lan} \mathrm{NE}$ & Langesse & Sols non cultivís & Sol de pâtruage ou de prainie $0-5 \mathrm{~cm}$ & Diamètre irféneưu à 2 xum & $24,04: 5001$ & 1,34 & 1,04 \\
\hline 15. $\tan \mathrm{NE}$ & Langesse & Sols non cultivés & Sol de pàtuage ou de prainie S-15.cm & Diamétre irfeñeư à 2 xum & $24,04: 2001$ & 1,16 & - \\
\hline 15./an NE & Langesse & Sols non cultivés & Sol de pâturaze ou de pramie $15-30.0 \mathrm{~m}$ & Diamètre irféneư à 2 mum & $24,04: 2001$ & 1,16 & - \\
\hline
\end{tabular}

Figure 5 - Exemple de tableau généré automatiquement.

Example of table automatically generated.

laboratoires de mesures. En effet, à l'heure actuelle, les envois d'échantillons aux différents laboratoires de mesures s'accompagnent de fiches papier sur lesquelles sont notées les informations descriptives, qui pourront à terme être contenues dans le code-barres. De même, et toujours dans un souci de traçabilité et de minimisation des risques d'erreur, une des prochaines amélioration prévue consiste à automatiser l'acquisition des saisies de données sur les pesées effectuées lors de la préparation des échantillons (report du poids, de la date de pesée, de la référence de la balance utilisée, ...). 